\title{
Cancer-associated fibroblasts exert a pro-angiogenic activity in Merkel cell carcinoma
}

Silvia Albertini ${ }^{1,2}$, Licia Martuscelli ${ }^{2}$, Cinzia Borgogna ${ }^{2}$, Sanamjeet Virdi ${ }^{3}$, Daniela Indenbirken ${ }^{3}$, Irene Lo Cigno ${ }^{2}$, Gloria Griffante ${ }^{2}$, Federica Calati ${ }^{2}$, Renzo Boldorini ${ }^{4}$, Nicole Fischer ${ }^{1 \#}$, Marisa Gariglio $^{2 \#}$

${ }^{1}$ Institute for Medical Microbiology, Virology and Hygiene, University Medical Center HamburgEppendorf, Hamburg, 20251, Germany.

${ }^{2}$ Virology Unit, Department of Translational Medicine, Novara Medical School, UPO, Novara, 28100 , Italy.

${ }^{3}$ Leibniz Institute for Experimental Virology, Technology Platform Next Generation Sequencing, Hamburg, 20251, Germany.

${ }^{4}$ Pathology Unit, Department of Health Sciences, Novara Medical School, UPO, Novara, 28100, Italy.

\# Corresponding authors:

Marisa Gariglio

Virology Unit, Department of Translational Medicine

Novara Medical School, Novara, Italy

Phone: +390321 660686

Email: marisa.gariglio@med.uniupo.ti

Nicole Fischer

Institute for Medical Microbiology, Virology and Hygiene

University Medical Center Hamburg-Eppendorf, Hamburg

Phone: +49-40-7410- 55171

Fax: $\quad+49-40-7410-53250$ 


\title{
Email: nfischer@uke.de
}

Running title CAFs promote angiogenesis in MCC

\begin{abstract}
The tumor microenvironment (TME) is a complex niche enveloping a tumor formed by extracellular matrix, blood vessels, immune cells, and fibroblasts constantly interacting with cancer cells. Although TME is increasingly recognized as a major player in cancer initiation and progression in many tumor types, its involvement in Merkel cell carcinoma (MCC) pathogenesis is currently unknown. Here, we provide the first molecular and functional characterization of cancerassociated fibroblasts (CAFs), the major TME component, in MCC patient-derived xenografts. We show that subcutaneous co-injection of patient-derived CAFs and human MCC MKL-1 cells into SCID mice significantly promotes tumor growth and metastasis. These fast-growing xenografts are characterized by areas densely populated with human CAFs, mainly localized around blood vessels. We also provide evidence that the growth-promoting activity of MCC-derived CAFs is mediated by the APA/Ang II-III/AT $\mathrm{R}_{1} \mathrm{R}$ axis, with the expression of aminopeptidase A (APA) in CAFs being the upstream triggering event. Altogether, our findings point to APA as a potential marker for MCC prognostic stratification and a novel candidate therapeutic target.
\end{abstract}

Keywords: angiogenesis / cancer associated fibroblasts / Merkel cell carcinoma / Merkel cell polyomavirus / tumor microenvironment

\section{Introduction}

Merkel cell carcinoma (MCC) is a highly aggressive skin cancer with an increased mortality rate compared to stage-matched melanoma. MCC mainly affects older and immunosuppressed patients who, at the time of diagnosis, often present with advanced clinical stage disease (Becker $e t$ al, 2017). The 5-year survival rate is dependent on the stage of metastasis and is approximately 
$51 \%$ in patients with a localized tumor, $35 \%$ in cases with loco regional metastasis, and as low as $15 \%$ in those with distant metastasis (Harms et al, 2016; Trinidad et al, 2019). The majority of MCCs, approximately $80 \%$ in the Northern Hemisphere, is caused by clonal integration of Merkel cell polyomavirus (MCPyV) into the host genome with persistent expression of the viral oncoproteins, small T-Antigen (sT) and large T-Antigen (LT) (Feng et al, 2008).

While virus positive (+) MCCs have relatively few genomic aberrations in the host genome and exhibit few mutations, virus negative (-) MCCs, on the other hand, have a high number of genomic aberrations and a predominant ultraviolet (UV) mutational signature (Goh et al, 2016; Gonzalez-Vela et al, 2017; Harms et al., 2016; Harms et al, 2015). Despite these notable differences in MCC etiology, both virus + and virus - MCCs are similar in presentation, prognosis, and response to therapy (Gonzalez-Vela et al., 2017).

MCPyV belongs to the family Polyomaviridae, which are highly prevalent, non-enveloped doublestranded DNA viruses causing opportunistic infections. MCPyV is the only human family member causally linked to tumorigenesis. Virus + MCCs show monoclonally-integrated replicationdefective forms of the viral genome with tumor hallmark mutations in the viral early gene region (Feng et al., 2008). Although MCPyV is clearly the etiologic agent of virus + MCCs, the correlations between the molecular phenotype, therapeutic approaches, and clinical outcome of MCPyV+ MCC remain poorly understood.

It is well established that tumor growth and progression are profoundly influenced by the tumor microenvironment (TME), which includes fibroblasts, inflammatory cells, blood vessels, extracellular matrix (ECM), and basement membranes (BMs) (Hinshaw \& Shevde, 2019). Specifically, the tumor populating fibroblasts_-termed cancer-associated fibroblasts (CAFs) —are desmoplastic cells that, once activated, contribute to cancer development through a variety of mechanisms. These include the release of various tumor-promoting factors, such as cytokines and chemokines, which, in turn, foster cancer cell growth and angiogenesis. CAFs are spindle-shaped, non-epithelial and non-immune cells embedded in the ECM that can be easily propagated in 
adherent cell cultures (Kalluri, 2016). Many reports have already extensively demonstrated that the desmoplastic stroma - mostly sustained by CAFs — in colorectal, breast, ovarian, head and neck cancers is associated with poor prognosis (Heichler et al, 2020; Karakasheva et al, 2018; Leung et al, 2018; Wen et al, 2019).

Despite the central role of CAFs in carcinogenesis, the function of CAFs in MCC pathogenesis and disease progression has been poorly investigated. Few publications on tumor infiltrating immune cells suggest a correlation between lymphocyte density, activation state, genetic diversity, and improved survival of MCC patients (Mendoza et al, 2020; Paulson et al, 2014; Samimi et al, 2019). A more recent study provided evidence of exosome transmitted cargo on the TME in MCC. Exosome transmitted miRNA-375 induced activation of $\alpha$ SMA-1, CXCL2, and IL$1 \beta$ genes, suggesting that horizontally transferred miR-375 is critical for polarizing fibroblasts toward a CAF phenotype in MCC (Fan et al, 2021).

To gain further insight into the functional role of the TME in MCC carcinogenesis, we have characterized the mechanism and functional role of MCC-derived CAFs - the major component of TME - in tumor growth and angiogenesis. Applying immunohistochemistry, transcriptomics, and functional assays, including tube formation and contraction assays, and in vivo tumorigenic assays, we show that patient-derived CAFs can differentially support MCC growth and angiogenesis through the renin-angiotensin system (RAS), specifically the APA/Ang II-III/AT 1 R axis, ultimately leading to increased angiogenesis and tumor progression. We also show that aminopeptidase A (APA) expression mainly occurs in fibroblasts, indicating a prominent role of CAFs in fostering a pro-angiogenic tumor microenvironment.

Overall, our findings provide the rationale for further evaluation of APA expression and function in MCC carcinogenesis as a potential marker of CAF-activated phenotype, which in turn may allow prognostic stratification of disease progression. In addition, pharmaceutical manipulation of this pathway may help develop future therapies against MCC. 


\section{Results}

\section{Characterization of MCC patients and corresponding CAFs}

We collected fresh tumor tissues from 9 MCC patients (Pts1-9) who underwent surgical excision of 3 primary tumors (pPts 4, 8 and 9), 5 local recurrences (rPts 1, 2, 3, 5, 7), and 1 lymph node metastasis (nPt 6). The clinicopathological features of the study cohort are summarized in Table 1.

Screening for MCPyV by both immunohistochemistry (IHC) (LT-antigen expression) and PCR (viral DNA detection) revealed that $6 \mathrm{MCCs}$ were MCPyV+(Pts 1, 2, 5, 6, 8, and 9), with viral loads ranging from 2 to 12 copies/cell (Table 1). In contrast, MCCs from Pts 3, 4, and 7 were MCPyV-. In a median follow-up period of 30 months, one patient, Pt3, died from MCC brain metastasis, three months after surgical excision of a relapsed MCC.

CAFs were successfully isolated from 8 of the aforementioned 9 MCC patients (Pts 2-9) by mechanic dissociation of the tumor masses. CAFs were named according to the stage of the relative tumor: primary (pCAFs); relapsed (rCAFs); or lymph node metastasis (nCAFs).

All patient-derived CAFs expressed, albeit to different extents, $\alpha \mathrm{SMA}-1$ and vimentin, hallmarks of activated fibroblasts and mesenchymal origin. In addition, CAFs proved negative for CD31 or cytokeratin 14 expression, excluding contamination by endothelial or epithelial cells (Figure 1A). In addition, all patient-derived CAFs, except for nPt6, showed enhanced extracellular matrix contractility compared to that of HFFs, as judged by an in vitro collagen contraction assay (Figure 1B). We next evaluated the mRNA expression levels of pro-inflammatory cytokines and chemokines known to be frequently upregulated in CAFs isolated from other carcinoma types (Kalluri, 2016). RT-qPCR revealed that IL-8, IL-6, CXCL12, and TGF- $\beta 1$ were generally upregulated in MCC-derived CAFs when compared to normal human foreskin fibroblasts (HFFs) (Figure 1C), with few notable exceptions: IL-8 in pPt8 and pPt9, and IL-6, CXCL12, and TGF- $\beta 1$ in $\mathrm{pPt} 9$.

To further characterize the stromal components of the original tumors, formalin fixed paraffin embedded (FFPE) tissue sections derived from primary and relapsed MCCs-if 
available - were analyzed by IHC using anti-vimentin (anti-Vim) staining. The overall density of $\mathrm{Vim}^{+}$cells varied significantly between samples, ranging from $10 \%$ to $60 \%$ [see Figure $2 \mathrm{~A}$ for representative panels of low density (pPt5) and high density (pPt6) stromal component]. Interestingly, we observed a slight increase in $\mathrm{Vim}^{+}$stromal components in the relapsed MCCs compared to the primary tumors (Figure 2B, grey pie chart), which led us to assess the localization of $\alpha \mathrm{SMA}-1^{+}$cells in the tumor stroma. Given the established regulatory association between CAFs and immune cells (Kalluri, 2016), we further aimed to determine the localization of $\mathrm{CD}^{+}$cytotoxic T cells and $\mathrm{CD}^{+} 8^{+}$macrophages in the desmoplastic stroma. As shown in Figure 2 and Figure EV1, $\alpha \mathrm{SMA}-1^{+}$cells were mainly localized in the stromal strakes and tumor edges, with only a few $\alpha \mathrm{SMA}-1^{+}, \mathrm{CD}^{+}$, and $\mathrm{CD}^{+} 8^{+}$cells penetrating the tumor nests. Within the stromal strakes, only the primary and relapsed specimens from Pt5 (Figure 2B) showed a higher proportion of CD8 ${ }^{+}$(red) and $\mathrm{CD}_{68}^{+}$(blue) cells over a background of more scattered $\alpha \mathrm{SMA}-1^{+}$cells (green). A substantial number of $\mathrm{CD}^{+} 8^{+}$was also observed in $\mathrm{Pt} 2, \mathrm{pPt} 3$, and $\mathrm{pPt}$. In all the other sections, the overall number of $\alpha \mathrm{SMA}-1^{+}$cells was higher and, for the most part, overlapped with the vimentin staining. Overall, the MCC stroma shows considerable variability in terms of cellular composition, density, and/or localization.

\section{Patient-derived CAFs differentially enhance tumor growth and metastasis formation when co-injected with MKL-1 cells into SCID mice}

To understand the impact of MCC-derived CAFs on tumorigenesis in vivo, we carried out xenograft mouse experiments by injecting CAFs together with tumor cells into an orthotopic site and directly assess the effects of CAFs on tumor growth (Albrengues et al, 2015). We employed an MCC xenograft metastasis mouse model that had been previously shown to develop spontaneous metastasis upon subcutaneous engraftment of the MCC cell lines, including MKL-1 (Knips et al, 2017). Patient-derived CAFs (Table 1) were subcutaneously inoculated into SCID mice $(\mathrm{n}=3)$ at a 1:1 ratio with $1 \times 10^{6}$ MKL-1 cells. Control experiments included the injection of MKL-1 cells 
alone $(n=3)$ or at a 1:1 ratio with HFFs $(n=3)$. With the exception of one out of three mice injected with CAFs from rPt5, which died soon after the injection for unrelated causes, mice were sacrificed at 3 months post-injection (pi) to ensure morphological and molecular characterization at a specific time point (Figure 3A). As shown in Figure 3A-C and EV2, tumors derived from co-injection of MKL-1 cells and rPt2, pPt4, or rPt5 CAFs exhibited the highest tumor growth rate and weight, while injection of MKL-1 together with rPt7 CAFs gave rise to intermediate-size tumors. However, when we co-injected MLK1 cells with CAFs derived from rPt3, nPt6, pPt8, or pPt9, we observed tumors with growth parameters similar to those obtained by injecting MKL-1 cells alone or with HFFs (Figure 3 A-C and Figure EV2).

We next assessed metastasis formation in different mouse organs (i.e., lung, brain, and bone marrow) by amplifying human Alu sequences, as previously published (Knips et al., 2017). Consistent with the tumor growth findings, the number of metastatic cells in the lungs of mice coinjected with MKL-1 cells and rPt2, pPt4, or rPt5 CAFs was significantly higher than that of mice injected with MKL-1 cells alone or in combination with HFFs or CAFs from rPt3, nPt6, rPt7, pPt8, or pPt9 (Figure 3D, left panel). In contrast, we only found few metastases in the brain and bone marrow in mice co-injected with MLK1 cells and CAFs from rPt3 or rPt5, respectively (Figure 3D middle and right panels). Of note, $\mathrm{Pt} 3$ died of brain metastasis three months after surgical removal of a relapsed MCC.

Overall, our results suggest that MCC-derived CAFs promote tumor progression and metastasis, although some degree of heterogeneity does exist among the patients analyzed.

\section{Enhanced tumor growth correlates with increased number of CAFs and angiogenesis in mouse xenografts}

To further characterize the differential ability of CAFs to promote tumor growth in our mouse xenografts, we measured CAF density, CAF distribution, and the extent of angiogenesis. For this purpose, we took advantage of an antibody that specifically recognizes human vimentin in IHC, as 
demonstrated by the lack of signal in xenografts derived from MKL-1 cells alone (Figure 4A, upper left panel). IHC revealed significant differences in fibroblast density among xenografts. In particular, the fast-growing xenografts derived from MKL-1 with $\mathrm{rPt} 2$, pPt4, rPt5, or rPt7 CAFs showed significantly increased $\mathrm{Vim}^{+}$staining compared with slow-growing xenografts (Figure 4A, left panels, and Figure 4B). Similarly, vessel number (Figure 4A, middle panels, and Figure 4C) and diameter (Figure 4D) were increased as shown by immunostaining for CD31. Serial sections were then co-stained by immunofluorescence with two antibodies, one specific for human Vim (green) and both, human and mouse Vim (red). This co-staining indicated that human fibroblasts (green) were accumulated in the fast-growing xenografts around the large blood vessels, in close contact with the murine endothelial cells of the vessel walls (red). Quantification of the number of vessels exhibiting this colocalization revealed that xenografts injected with MKL-1 cells plus rPt2, pPt4, rPt5, or rPt7 CAFs showed a high percentage of vessels surrounded by large (black) or medium (dark gray) numbers of human fibroblasts. By contrast, xenografts injected with control cells or with MKL-1 plus pPt8 or pPt9 CAFs mainly displayed vessels surrounded by only a small number of human fibroblasts (light gray, Figure 4E). Our data point to a positive correlation between the percentage of human fibroblasts surrounding the vessels and the vessel diameter (Figure 4F).

The transcriptome profile of the xenografts derived from growth-promoting CAFs is characterized by a pro-angiogenic signature

In order to unravel CAF-mediated functions in promoting tumor formation, we performed bulk transcriptome sequencing (RNA-Seq) of xenografts obtained by injecting MKL-1 cells with CAFs from $\mathrm{rPt} 2$, $\mathrm{pPt} 4$, or $\mathrm{rPt5}$, which we had previously shown to promote enhanced tumor formation (Figure 3). As negative controls (CTRLs), we injected mice with MKL-1 cells alone or with HFFs, which were treated as biological duplicates based on principle component analysis (Appendix Figure S1). Differential gene expression analysis of CAFs vs CTRLs revealed 234 differentially expressed genes (DEGs), of which $98 \%$ were upregulated $(n=227)$ and only $2 \%$ downregulated $(n$ 
= 3; Figure 5A-B) [false discovery rate $(\mathrm{FDR})<0.05 ; \log 2 \mathrm{FC}>1$ ]. Functional annotation of DEGs (Figure 5C and Appendix Table S1) showed significant enrichment for angiogenesis-related biological processes, such as blood vessel development and morphogenesis and vasculature development. Furthermore, gene set enrichment analyses (GSEA) of the DEGs involved in the top 10 annotated gene ontology (GO) terms (Figure 5C and Appendix Table S1) showed a positive normalized enrichment score (NES) and a significant FDR $<0.25$ for all the gene set lists analyzed, thereby confirming their distribution in the top portion (upregulated) of the gene dataset (Appendix Figure S2). Biological pathway enrichment analysis was also performed through KEGG pathway analysis with DEGs being highly associated with pathways defined as PI3K-Akt signaling pathway and ECM-receptor interaction (Figure 5D), which have been previously linked to MCC carcinogenesis and CAF functions in tumorigenesis (Hafner et al, 2012; Kalluri, 2016; Nardi et al, 2012).

To broaden our analysis, we investigated the DEGs using ingenuity pathway analysis (IPA). IPA analysis predicted a wide range of activated and decreased disease and function in CAFs compared to CTRLs (Figure EV3 and Appendix Table S2). More specifically, 68 disease and function annotations were defined as being activated (Figure EV3, orange bars), including growth, migration, and invasion of tumor cells, development of vasculature, and angiogenesis, while 16 annotations displayed a decreased activation state (blue bars), including necrosis, apoptosis, and cell death of carcinoma cell lines.

Overall, the transcriptome results from the xenografts injected with tumor-promoting CAFs support the involvement of CAFs in tumor development and progression and, in particular, suggest their engagement in angiogenesis processes in MCC.

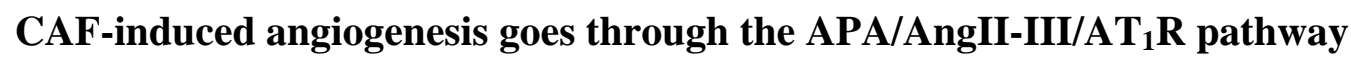

To determine the functional role of the pro-angiogenic signature revealed by the transcriptome analysis, we first assessed the expression levels of the top 10 upregulated genes (Table 2) by RT- 
qPCR. In particular, we focused our attention on the arginine vasopressin receptor 1A $(A V P R I A)-$ here the most upregulated one- previously shown to play a pivotal role in prostate cancer progression and metastasis formation (Zhao et al, 2019), and on the glutamyl aminopeptidase $(E N P E P)$ and membrane metalloendopeptidase $(M M E)$ genes, which were among the GO term angiogenesis genes listed in Appendix Table S1. Quantitative RT-PCR analysis, performed using total RNA re-extracted from all the xenografts - with the exclusion of pPt4 and pPt8 CAF-injected xenografts due to insufficient RNA quality—, revealed that all three genes were upregulated, albeit to different extents, in the xenografts co-injected with MKL-1 and CAFs compared to MKL-1 alone or MKL-1 plus HFFs (Figure 6A). Importantly, their expression levels were significantly higher in MKL-1/rPt2, rPt5, or rPt7 CAF tumors than in MKL-1/rPt3, nPt6, or pPt9 tumors, suggesting a correlation between upregulation of $A V P R 1 A, E N P E P$ and $M M E$ and increased tumor burden. Of note, AVPRIA, ENPEP and MME expression levels also correlated with the number of $\mathrm{Vim}^{+}$cells in the xenografts (Figure 6B). While in MKL-1 cells ENPEP, MME, and AVPRIA were all transcriptionally inactive, ENPEP and $M M E$ were expressed in HFFs and patient-derived CAFs (Figure 6C). Interestingly, AVPRlA gene expression was only detected in xenografts (Figure 6A) but not in the 2D cell cultures of all the cell lines and primary cells analyzed.

ENPEP encodes the membrane aminopeptidase A (APA), a membrane-bound metallopeptidase that converts bioactive peptides such as angiotensin II (Ang II) into angiotensin III (Ang III) (George et al, 2010). Both peptides are known to exert pro-angiogenic activity via the angiotensin II type 1 receptor $\left(\mathrm{AT}_{1} \mathrm{R}\right)$ (Bosnyak et al, 2011; George et al., 2010; Reaux et al, 2001). In addition, Ang II conversion into Ang III prevents Ang II cleavage into Ang(1-7) by ACE2, which in turn binds the MAS receptor, promoting an anti-angiogenic effect (Catarata et al, 2020). Thus, we decided to test whether pharmacological inhibition of this pathway would impair CAFinduced angiogenesis in an in vitro model of tubulogenesis. Human dermal blood endothelial cells (HDBECs) were grown on a monolayer of patient-derived CAFs or HFFs, and their ability to form tubule-like structures was assessed after 10 days. All patient-derived CAFs supported in vitro tube 
formation, whereas non-activated HFFs failed to generate tubular structures (Figure EV4). For all subsequent in vitro tube formation assays, we used rPt-5 CAFs due to their robust tumor growthpromoting and angiogenic properties. The APA inhibitor amastatin was used at concentrations of 1 , 10, and $100 \mu \mathrm{M}$. Lack of cell toxicity was assessed by MTT assay (Appendix Figure S3). As shown in Figure 7 and Figure EV5, when amastatin was added to the co-cultures, we observed a dosedependent inhibition of tubule-like structure formation, measured as mean tubule length, total tubule length, and tubule density upon CD31 staining (Figure 7A-B). Similarly, tube formation was inhibited when the co-cultures were treated with the $\mathrm{AT}_{1} \mathrm{R}$ inhibitor candesartan (Figure 7C-D and Figure EV5) (Uemura et al, 2008). We did not observe a synergistic effect when co-cultures were treated simultaneously with the two inhibitors (Figure EV5).

Altogether, these findings clearly indicate that the APA/AT $T_{1}$ pathway is involved in the pro-angiogenic activity of MCC-derived CAFs.

\section{Discussion}

There is growing evidence that cancer progression is supported by changes in the tumor microenvironment, where CAFs are the most abundant cell type (Kalluri, 2016). While the protumorigenic role of CAFs in various solid tumors has been clearly established, the function of these cells in MCC initiation and progression remains poorly understood. The present study describes a novel mechanism of MCC etiopathogenesis based on CAF-driven tumor growth and angiogenesis via the APA/Ang II-III/AT ${ }_{1} \mathrm{R}$ axis, in which APA expression in CAFs potentially accounts for this major contribution.

Our molecular analysis shows that MCC-derived CAFs are characterized by increased aSMA-1 expression as well as enhanced contractility and secretion of pro-inflammatory cytokines, hallmarks of an activated phenotype. Furthermore, 6 out of the 9 MCCs analyzed in our study were positive for MCPyV, which together with UV light is the main etiologic factor for MCC (DeCaprio, 2021). Although we did not find any specific correlations between CAF activity and/or phenotype 
and MCPyV positivity, most of these MCC-derived CAFs, in contrast to non-activated fibroblasts, significantly enhanced tumor growth of MKL-1 cells in immunosuppressed mice. Notably, xenografts generated by co-injection of rPt2, pPt4, rPt5, or rPt7 CAFs and MKL-1 cells revealed increased tumor volume and weight compared with xenografts generated with rPt3, nPt6, pPt8, or pPt9 CAFs plus MLK-1 cells. Consistent with an active role of CAFs in enhancing tumor progression, an increased number of lung metastases were observed in mice injected with $\mathrm{rPt}$, pPt4, or rPt5 CAFs plus MKL-1 cells. Another interesting finding is that we could only detect brain metastases in mice injected with CAFs derived from Pt3, who had died from brain metastasis three months after the surgical resection of the tumor. Although this was only one case, the apparent correlation between CAF-driven brain metastasis and poor disease outcome further strengthens the impact of CAFs on MCC progression.

An important consideration from our in vivo tumorigenicity assays is that patient-derived CAFs may differentially affect MCC growth and metastasis, implying that their in vivo acquired activated phenotype along with their intrinsic heterogeneity in driving tumor growth and metastasis are fully preserved in our culture conditions. More specifically, the observed enhanced xenograft growth correlated with increased stromal cells density in the xenografts, as documented by the quantification of $\mathrm{Vim}^{+}$cells in tissue sections. Of note, we found that $\mathrm{Vim}^{+}$cells were more abundant in fast-growing than in slow-growing xenografts, and that in the latter co-injection of CAFs did not significantly increase tumor growth induced by MKL-1 cells alone. Consistent with this observation, the number of human fibroblasts detectable in the xenografts where normal fibroblasts were co-injected with MKL-1 cells was extremely low. The observed increased CAF density was accompanied by augmented vessel density and size, suggesting that CAFs may actively contribute to vascularization, favoring tumor growth. Another interesting observation supporting the pro-angiogenic function of CAFs is that the areas near the blood vessels in the rapidly growing xenografts were densely populated by human fibroblasts. 
Comparative gene expression profiling by whole genome RNA-sequencing of xenografts injected with the growth-promoting $\mathrm{rPt} 2$, pPt4, or $\mathrm{rPt} 5 \mathrm{CAFs} v s$ xenografts injected with HFFs or MKL-1 cells alone showed significant enrichment in angiogenesis-related biological processes, such as blood vessel development and morphogenesis and vasculature development. Among the top ten most upregulated genes, the mRNA levels of AVPRIA, ENPEP and MME were significantly increased in fast-growing $v s$ slow-growing xenografts. The fact that $M M E$ and $E N P E P$ expression was increased in CAFs vs HFFs and totally absent in MKL-1 cells cultured as monolayers strongly suggests that CAFs are the major site of their expression. Intriguingly, while AVPRlA gene expression was confirmed at the RNA level in both xenografts (Figure 5E) and patient-derived MCC specimens (Appendix Figure S4), we failed to detect its expression in both HFFs/CAFs and MKL-1 cells grown in vitro as either monolayer or co-cultures. Thus, further experiments in vivo are clearly needed to ascertain whether AVPR1A is induced in CAFs or tumor cells and plays a role in MCC progression and metastasis.

ENPEP encodes APA, a homodimeric type II membrane-spanning cell surface protein with a zinc metallopeptidase activity that hydrolyzes $\mathrm{N}$-terminal glutamyl or aspartyl residues from oligopeptide substrates (Nanus et al, 1993). The best understood role of APA is the conversion of angiotensin II (Ang II) to angiotensin III (Ang III) in the renin-angiotensin system (RAS) (Nanus et al., 1993). Most Ang II and Ang III actions involve $\mathrm{AT}_{1} \mathrm{R}$, a G protein-coupled receptor, which is upregulated in many cancer types (Bosnyak et al., 2011; George et al., 2010). In the brain, experiments involving APA and APN specific inhibitors have provided clear evidence that Ang III is the main effector peptide involved in $\mathrm{AT}_{1} \mathrm{R}$ activation and vasopressin release (Zini et al, 1996). In addition, the blockage of Ang II conversion to Ang III with APA inhibitors has been shown to favor the activation of other metabolic pathways, such as that involving the angiotensin-converting enzyme 2 (ACE2) and the Mas receptor ligand, whose activity is known to inhibit cancer growth and angiogenesis (Reaux et al., 2001; Wright et al, 2012). 
Although RAS was originally discovered for its relevant role in regulating vascular homeostasis, emerging data from in vitro, animal, and clinical studies indicate that aberrant regulation of this pathway can promote tumor growth and angiogenesis in several tumor types (George et al., 2010). Conversely, RAS inhibition can suppress tumor growth, metastasis, and angiogenesis in a variety of tumor models (Pinter \& Jain, 2017). Fittingly, APA is upregulated in the perivascular cells of angiogenic tumor vessels where it promotes angiogenesis, while in normal blood vessels it is only barely detectable (Marchio et al, 2004).

In this study, we show that the ENPEP gene encoding APA is transcriptionally upregulated in fast-growing xenografts where it plays a crucial role in CAF-induced angiogenesis. We also demonstrate that APA inhibition with the antagonist amastatin impairs tubule formation. This inhibitory effect is dose-dependent and primarily affects the length of tubule-like structures as well as their extension and density rate, indicating that the tubulogenesis activated by the CAF co-culture strictly relies on APA activity. Given that the two enzymatic byproducts of APA, Ang II and Ang III, mainly function through $\mathrm{AT}_{1} \mathrm{R}$, it is not surprising that treatment of $\mathrm{CAF} / \mathrm{HDBEC}$ co-cultures with the $\mathrm{AT}_{1} \mathrm{R}$ inhibitor candesartan impaired the formation of branched, interconnecting networks of endothelial cell tubules, as we have demonstrated in amastatin-treated cultures.

Given that very few studies have thoroughly addressed the biological significance of stromal cells in MCC progression, our investigation is the first to provide evidence that these cells may play a relevant role in modulating tumor growth, particularly angiogenesis. This new paradigm would be consistent with a recent report showing that intercellular communication between MCC cells and stromal fibroblasts is required for the generation of activated/polarized CAFs (Fan et al., 2021). Consistent with our findings, variability/heterogeneity of stromal components populating MCC lesions has been reported, a phenomenon also observed in many other cancers. This aspect is particularly important considering that, to date, no reliable prognostic markers for MCC have been identified. Thus, it is tempting to speculate that quantification/characterization of the stromal components in MCC may be used to predict more accurately disease progression. 
In conclusion, our study highlights the biological role of CAFs in MCC development and metastasis formation. The growth-promoting activity of MCC-derived CAFs is mostly due to increased angiogenesis. Mechanistically, we demonstrate that this process is largely mediated by the APA/Ang II-III/AT $\mathrm{R}_{1} \mathrm{R}$ axis, with APA expression in CAFs being the upstream triggering event, suggesting a potential role of APA as a prognostic and therapeutic marker for MCC. Further experiments will be necessary to determine the effect of APA inhibitors, alone or in combination with chemotherapy, on tumor growth, angiogenesis, and metastasis formation in vivo. 


\section{Materials and Methods}

\section{Patients and cell culture}

Nine patients with a histological diagnosis of Merkel cell carcinoma (MCC) who underwent surgical excision of primary or relapsed tumor or lymph nodal metastasis were included in this study. They were recruited between July 2015 and April 2019 through "Rete Oncologica Piemontese" from various Hospitals in the Piedmont region (Northern Italy). All patients were informed at the time of the surgery that their clinical data and specimens (i.e., FFPE blocks and fresh tissue) would be used for research purposes and signed a written informed consent form. Study approval was obtained from the Ethics Committee of "Maggiore della Carità" Hospital (agreement number 213/CE). Fresh human MCC specimens were obtained following surgical excision of three primary tumors, four relapsed tumors, and one lymph node metastasis.

CAFs were directly isolated from the patient tissue as previously described (Lau et al, 2016). Briefly, tumors were washed twice with PBS, visible excess of fat tissue was removed, and tissues were finely minced using a sterile scalpel. Homogenized tissues were incubated in RPMI media supplemented with $10 \% \mathrm{FBS}$ and $\mathrm{P} / \mathrm{S}$ and maintained at $37^{\circ} \mathrm{C}$ in $5 \% \mathrm{CO}_{2}$. After $24 \mathrm{~h}$, the unattached cells were removed, and the remaining cells were allowed to grow on the plate for 23 weeks with the media replenished every other day. When adherent cells reached confluence, they were trypsinized and transferred into a new culture flask and grown in DMEM supplemented with $10 \%$ FBS and P/S. For functional assay, only low passage numbers were used.

The MCPyV-positive MCC cell line MKL-1 (Rosen et al, 1987) was purchased from Sigma-Aldrich (Cat. No. 09111801) and grown in RPMI 1640 (Gibco) supplemented with 10\% fetal bovine serum (FBS, Sigma-Aldrich, Milan, Italy), $100 \mathrm{U} / \mathrm{ml}$ penicillin, and $0.1 \mathrm{mg} / \mathrm{ml}$ streptomycin (P/S). HFFs (ATCC Cat. No. SCRC-1041) were maintained in DMEM 10\% FBS and P/S. HDBECs (PromoCell, Cat. No. C-12211) were grown in endothelial cell basal medium (PromoCell, Heidelberg, Germany) supplemented with Endothelial Cell GM MV 2 Supplement Pack (PromoCell, Heidelberg, Germany). 


\section{Collagen contractility assay}

Collagen contractility assay was performed as previously described (Saraswati et al, 2019). Briefly, $3 \times 10^{5}$ HFFs or CAFs were embedded in $750 \mu$ l of rat tail collagen-I (Corning, Germany) supplemented with $1.5 \mathrm{ml}$ of serum-free DMEM and $12.5 \mu \mathrm{l}$ of $1 \mathrm{~N} \mathrm{NaOH}$. Six-hundred $\mu$ l of cell/collagen mix was plated in 24-well plates and incubated for 15-20 min until gelled. The gel was then separated from the walls of the well with the help of a $30 \mathrm{G}$ needle, and $600 \mu \mathrm{l} / \mathrm{well}$ of DMEM supplemented with $10 \%$ FBS was added on top of the gel. Gels were incubated for $24 \mathrm{~h}$ at $37^{\circ} \mathrm{C}$, fixed with 4\% PFA, and stained with eosin. Well and gel diameters were measured using Fiji (Schindelin et al, 2012). The percentage of gel contraction was calculated using the formula $100 \mathrm{x}$ (well diameter - gel diameter)/well diameter.

\section{Immunofluorescence, immunohistochemistry, and immunoblotting}

Consecutive 5- $\mu \mathrm{m}$ thick tissue sections were obtained from FFPE blocks. Hematoxylin and eosin (H\&E) and immunohistochemistry staining were performed using the automated immunostainer BenchMark ULTRA Stainer (Ventana Medical System, Tucson, AZ). For immunofluorescence, antigen unmasking was performed by heating the slides in a conventional decloaking chamber at $750 \mathrm{~W}$ for $15 \mathrm{~min}$, followed by an additional step at $350 \mathrm{~W}$ for $10 \mathrm{~min}$ in $10 \mathrm{mM}$ citrate buffer at $\mathrm{pH}$ 6.0 (Vector Laboratories, Burlingame, CA, USA). Primary antibodies were diluted together in 5\% normal goat serum/PBS and incubated $\mathrm{ON}$ at $4^{\circ} \mathrm{C}$. Tissue images were acquired using Panoramic Midi Scanner (3D-Hystec) and staining was quantified using QuPath software (Bankhead et al, 2017).

For immunoblotting, whole-cell protein lysates were prepared and processed as previously described (Gugliesi et al, 2005). Anti- $\beta$-actin antibodies (Mab) (Active Motif) were used as control for protein loading. Immunocomplexes were detected using sheep anti-mouse or donkey anti-rabbit immunoglobulin secondary antibodies conjugated to horseradish peroxidase (HRP) (GE Healthcare Europe $\mathrm{GmbH}$ ) and visualized by enhanced chemiluminescence (Super Signal West Pico; Thermo 
Fisher Scientific) according to the manufacturer's instructions. Images were acquired using Quantity One software (version 4.6.9; Bio-Rad Laboratories Srl). The antibodies used in this study are listed in Appendix Table S3.

\section{Quantitative nucleic acid analysis}

MCC tumor total DNA was isolated with a QIAamp DNA Mini Kit (QIAGEN). To determine MCPyV viral load in MCC specimens, quantitative PCR (qPCR) was performed using $500 \mathrm{nM}$ primers and SsoAdvanced Universal SYBR Green Supermix (Bio-Rad). The reaction conditions consisted of a $30-\mathrm{s} 95^{\circ} \mathrm{C}$ enzyme-activation cycle, 40 cycles $(10 \mathrm{~s})$ of denaturation at $95^{\circ} \mathrm{C}$, and 10 s of annealing at $60^{\circ} \mathrm{C}$. Copy number analysis was completed by comparing the unknown samples with standard curves of linearized MCPyV DNA. The GAPDH DNA copy number was used as endogenous control.

Total RNA was extracted from CAFs, xenografts, and MCCs using TRIzol (Thermo Fisher Scientific, Inc.), and $1 \mu \mathrm{g}$ per sample was retrotranscribed using iScript cDNA synthesis kit (BioRad Laboratories Srl). To detect cellular gene expression, reverse-transcribed cDNAs were amplified in duplicate using SensiFast SYBR (Bioline). Real-time quantitative reverse transcription (qRT)-PCR analysis was performed on a CFX96 real-time system (Bio-Rad Laboratories Srl) under the following conditions: $95^{\circ} \mathrm{C}$ for $10 \mathrm{~min}$, followed by 40 cycles at $95{ }^{\circ} \mathrm{C}$ for $15 \mathrm{~s}$, and $60{ }^{\circ} \mathrm{C}$ for 1 min. Relative quantification [comparative $\mathrm{Ct}(\Delta \Delta \mathrm{Ct})$ method] was used to compare the expression level of the tested genes with the housekeeping gene glyceraldehyde-3-phosphate dehydrogenase (GADPH).

To detect disseminated tumor cells in mouse organs, total DNA was extracted from the organs of the engrafted mice using QIAamp DNA Mini Kit (Qiagen), and 40 ng were amplified using human specific Alu sequences as previously described (Knips et al., 2017; Nehmann et al, 2010). Amplification was performed with a CFX96 real-time system (Bio-Rad Laboratories Srl) under the following conditions: $10 \mathrm{~min}$ at $95 \mathrm{C}$, followed by 50 cycles at $95{ }^{\circ} \mathrm{C}$ for $5 \mathrm{~s}, 67^{\circ} \mathrm{C}$ for $5 \mathrm{~s}$, 
and $72{ }^{\circ} \mathrm{C}$ for $20 \mathrm{~s}$. Quantification of human metastatic cells in mouse organs was determined by using standard curves generated with MKL-1 DNA dilutions ranging from 1 to $10^{6}$ cells $/ \mathrm{ml}$. The number of metastatic cells was normalized to the ng of DNA used for the amplification.

The QX200 Droplet Digital PCR System (Bio-Rad Laboratories) was used for absolute quantification of mRNA expression in MCC tumor. For each assay, a reaction mixture of $22 \mu \mathrm{L}$ was prepared with $11 \mu \mathrm{L}$ EvaGreen SuperMIX (Bio-Rad Laboratories), $1.1 \mu \mathrm{L}$ of $10 \mu \mathrm{M}$ of each primer, and $5 \mathrm{ng}$ of cDNA. For droplet generation, $20 \mu \mathrm{L}$ reaction mix was used, and the droplets were transferred to a 96-well plate. Samples were amplified in a C1000 Touch Thermal Cycler (Bio-Rad Laboratories) according to the following protocol: $95{ }^{\circ} \mathrm{C}$ for $5 \mathrm{~s}, 40$ cycles of $95{ }^{\circ} \mathrm{C}$ for $30 \mathrm{~s}$ and $58{ }^{\circ} \mathrm{C}$ for $60 \mathrm{~s}$, followed by $4^{\circ} \mathrm{C}$ for $5 \mathrm{~s}, 90{ }^{\circ} \mathrm{C}$ for $10 \mathrm{~min}$, and $4{ }^{\circ} \mathrm{C}$ for $40 \mathrm{~s}$. Data were analyzed using QuantaSoft (Bio-Rad Laboratories). PCR primer sequences are detailed in Appendix Table S4.

\section{In vivo tumorigenic assay}

Female SCID mice (CB17/lcr-Prkdc scid/lcrlcoCrl; Charles River Laboratories, Sulzfeld, Germany) were housed under pathogen-free conditions in our animal facilities in accordance with "The Guide for the Care and Use of Laboratory Animals", and the experimentation was approved by the Italian Ministry of Health (Agreement number 231/2020-PR). HFFs or CAFs from Pt2 to 9 were injected along with MKL-1 cells as previously described (Knips et al., 2017; Lau et al., 2016). Briefly, $50 \mu 1$ of matrigel (Corning) suspension containing 1 × $10^{6}$ (MKL-1 alone) or $2 \times 10^{6}$ cells $\left(1 \times 10^{6}\right.$ MKL-1 combined with $1 \times 10^{6}$ HFFs or CAFs) were injected subcutaneously into 6-week-old SCID mice. All mice were observed and examined weekly, and tumor volumes were evaluated by measuring two perpendicular diameters of the tumor with a caliper. Individual tumor volume was calculated as $(V)=a \times b^{2} / 2$, with $a$ being the major and $b$ the minor diameter. Mice were sacrificed by cervical dislocation after 3 months from the injection. For each mouse, primary tumor, lungs, liver, brain, bone marrow, and renal and lumbar aortic lymph nodes were collected. Primary tumors were 
halved, and half of them were used to extract total RNA, while the other was fixed in $10 \%$ neutralbuffered formalin and embedded in paraffin blocks.

\section{RNAseq analysis and bioinformatic analysis}

Total RNA from the explanted xenografts was isolated using TRIzol (Thermo Fisher Scientific, Inc.), and RNA integrity was analyzed with the RNA 6,000 Nano Chip on an Agilent 2100 Bioanalyzer (Agilent Technologies). rRNA was depleted using the RiboCop rRNA Depletion Kits (Lexogen), and RNA-Seq libraries were generated using the CORALL Total RNA-Seq Library Prep Kit (Lexogen) according to manufacturer's instructions. Size and quality of the libraries were assessed using a BioAnalyzer High Sensitivity Chip (Agilent). All samples were normalized to 2 $\mathrm{nM}$ and pooled equimolar. The library pool was sequenced on the NextSeq500 (Illumina) with 1x75bp, with 21 to 31 mio reads per sample. Gene expression was calculated by means of gene annotations from ENSEMBL (version 98) for human GRCh38 genome assembly after aligning raw FASTQ files using STAR (2.7.0f) (STAR https://pubmed.ncbi.nlm.nih.gov/23104886/) with default parameters with option “--quantMode GeneCounts”. Differential gene expression analysis was performed using DESeq2 package (Love et al, 2014). Null variance of Wald test statistic output by DESeq2 was re-estimated using the R-package FDR-tool (Strimmer, 2008) to re-calculate $P$ values - and adjusted using Benjamini-Hochburg method-for the final list of differentially expressed genes.

Gene ontology enrichment analysis was performed on the obtained gene list using topGO (Alexa \& Rahnenfuhrer, 2019). Ten non-DE-genes having similar mean expression level per each DE-gene were selected for enrichment analysis as background using $\mathrm{R}$ package genefilter (https://www.rdocumentation.org/packages/genefilter/versions/1.54.2). GO plots for enrichment of biological process were plotted using R package ClusterProfiler (Yu et al, 2012). The list of DEGs involved in the top $10 \mathrm{GO}$ terms was further analyzed by GSEA version 3.0 software (Broad Institute). Preranked analyses were performed by sorting differentially expressed genes according to 
their fold changes without prior filtering on significance or effect size. Enrichment map was used for visualization of the GSEA results. One thousand permutations were performed for each analysis.

Disease and function bioinformatics analysis were generated through the use of IPA (QIAGEN Inc., https://www.qiagenbioinformatics.com/products/ingenuitypathway-analysis). The score is generated based on hypergeometric distribution, where the negative logarithm of the significance level is obtained by Fisher's exact test at the right tail. The $-\log (P$-value $)>2$ was adopted as threshold, the Z-score $>2$ was defined as the threshold of significant activation, while the Z-score $<-2$ was defined as the threshold of significant inhibition.

\section{Tube formation assay}

The in vitro tube formation assay was performed as previously described (Hetheridge et al, 2011). Briefly, $3 \times 10^{4}$ CAFs or HFFs were seeded on $10 \mathrm{~mm}$ glass coverslips (Thermo Fisher Scientific, Inc.) and, when they reached confluence, covered by a layer of HDBECs $\left(1 \mathrm{x} 10^{4}\right)$. The medium (ECGM-2 w/o VEGF) was replenished every other day. When the cells were treated with APA or $\mathrm{AT}_{1} \mathrm{R}$ pharmacological inhibitors, the medium was refreshed $24 \mathrm{~h}$ after HDBEC plating and supplemented with the indicated concentration of amastatin (A1276; Sigma-Aldrich), candesartan (CV11974; Tocris), or vehicle (DMSO or MeOH respectively) every other day. After 10 days of coculture, cells were fixed with $4 \% \mathrm{PFA} / \mathrm{PBS}$ for $10 \mathrm{~min}$ at $4^{\circ} \mathrm{C}$, washed with PBS, and permeabilized with $0.05 \%$ Tween 20 in PBS. Samples were blocked with 3\% BSA in PBS before incubation with the primary antibody $\alpha \mathrm{CD} 31$ (BD Pharmingen). Images were acquired using SP5 confocal microscope (Leica). The mean tubule length was quantified using Fiji software (Schindelin et al., 2012). Tubule density and total tubule length were quantified using WimTube software program (Wimasis, Munich, Germany).

\section{Cell viability assay}


Cells were seeded at a density of $1 \times 10^{4} /$ well in a 96-well culture plate for $24 \mathrm{~h}$ and then treated with the indicated concentrations of amastatin, candesartan, or vehicle $(\mathrm{MeOH}$ or DMSO, respectively). Forty-eight h later, cell viability was determined using the 3-(4,5-dimethylthiazol-2yl)-2,5-diphenyltetrazolium bromide (MTT) (Sigma-Aldrich, Milan, Italy) according to the manufacturer's instruction.

\section{Statistical analysis}

All statistical tests were performed using GraphPad Prism version 7.00 for Mac (GraphPad Software). The data are presented as means \pm standard deviations (SD). For comparisons consisting of two groups, means were compared by two-tailed Student's t test. For comparison among three or more groups, means were compared using one-way or two-way ANOVA with Bonferroni posttest. Differences were considered statistically significant at a $P$-value of $<0.05$. 


\section{Acknowledgments}

We thank Marcello Arsura for critically reviewing the manuscript and Bernd Zobiak and Antonio Virgilio Failla, HEXT, UKE Microscopic Imaging Facility (UMIF) for their help with the confocal microscopy. We thank Kerstin Reumann and Christina Herrde for excellent technical support with the NGS library preparation and sequencing. We are very grateful to "Rete Oncologica Piemontese" for providing the MCC specimens. The authors gratefully acknowledge Michela Salvo from the Histology Research Core Facility at University of Piemonte Orientale (Novara, Italy) for technical support in tissue processing and histological analysis and Valeria Caneparo at CAAD - Center for Translational Research on Autoimmune and Allergic Disease (Novara, Italy) for assistance with ddPCR assays.

This work was supported by grants from the Italian Ministry for University and Research-MIUR (PRIN 2017) to C.B., the AGING Project—Department of Excellence-DIMET to M.G., and the Università del Piemonte Orientale, and "Rete Oncologica Piemontese" to R.B. and M.G.. S.A. was supported by the Rotary Global Grant (2019-2020), which was sponsored by the Rotary Club of Novara and financed by the Rotary Foundation and University of Piemonte Orientale, Department of Excellence - DIMET, and by the Erich und Gertrud Roggenbuck Foundation, Hamburg (20202021).

\section{Author contribution}

Conception and design: SA; NF; MG. Development and methodology: SA; LM; CB; NF; MG. Acquisition of data: SA; LM; CB; SV; DI; GG; FC; NF; MG. Analysis and interpretation of data: SA; LM; CB; SV; DI; ILC; RB; NF; MG. Administrative, technical, or material support: SA; SV; DI; ILC; GG; FC; RB; NF; MG. Writing, review, and/or revision of the manuscript: SA; LM; CB; NF; MG. Final approval: All authors.

\section{Conflict of Interest}


The authors declare no conflict of interest.

\section{The paper explained:}

\section{Problem}

Merkel cell carcinoma (MCC) is a highly aggressive skin cancer, with most patients presenting with already advanced tumor stages at the time of diagnosis. The 5-year survival rate significantly drops with the presence of metastases. While immune therapy shows partial regression of MCC in the majority of cases, a significant proportion of patients develop immunotherapy-resistant tumor cells and even hyperprogressive disease courses. Analyzing the tumor microenvironment (TME), in particular the contribution of cancer-associated fibroblasts (CAFs) - the major component of TME - will significantly contribute to our understanding of MCC progression and might be helpful to identify new therapeutic targets.

\section{Results}

This study describes the molecular and functional characterization of CAFs in MCC. CAFs were isolated from primary or recurrent MCC tumors and functionally characterized by gene expression and contractility assays. Their impact on MCC tumorigenesis was investigated in a xenograft mouse model. These experiments, together with transcriptome analyses of the xenografts obtained by subcutaneous co-injection of patient-derived CAFs and human MCC MKL-1 cells, demonstrate that the growth-promoting activity of CAFs is mediated by the APA/Ang II-III/AT 1 R axis, with expression of aminopeptidase A (APA) being the upstream triggering event.

\section{Impact}

In this study, we have characterized, for the first time, the role of CAFS in MCC tumor progression. We demonstrate that those CAFs that exhibit tumor-promoting activity display high expression levels of aminopeptidase A (APA). Furthermore, we show a positive correlation of APA expression with CAF localization around blood vessels and enhanced tumor angiogenesis. These findings decipher APA and the APA/Ang II-III/ATR ${ }_{1} \mathrm{R}$ axis as a putative signaling pathway amenable to 
bioRxiv preprint doi: https://doi.org/10.1101/2021.07.30.454425; this version posted August 3, 2021. The copyright holder for this preprint (which was not certified by peer review) is the author/funder. All rights reserved. No reuse allowed without permission.

therapeutic intervention, especially since there are known inhibitors of this pathway already approved for other diseases.

\section{References}

Albrengues J, Bertero T, Grasset E, Bonan S, Maiel M, Bourget I, Philippe C, Herraiz Serrano C, Benamar S, Croce O et al (2015) Epigenetic switch drives the conversion of fibroblasts into proinvasive cancer-associated fibroblasts. Nat Commun 6: 10204

Alexa A, Rahnenfuhrer J. (2019) topGO: Enrichment Analysis for Gene Ontology. R package version 2.36.0.

Bankhead P, Loughrey MB, Fernandez JA, Dombrowski Y, McArt DG, Dunne PD, McQuaid S, Gray RT, Murray LJ, Coleman HG et al (2017) QuPath: Open source software for digital pathology image analysis. Sci Rep 7: 16878

Becker JC, Stang A, DeCaprio JA, Cerroni L, Lebbe C, Veness M, Nghiem P (2017) Merkel cell carcinoma. Nat Rev Dis Primers 3: 17077

Bosnyak S, Jones ES, Christopoulos A, Aguilar MI, Thomas WG, Widdop RE (2011) Relative affinity of angiotensin peptides and novel ligands at AT1 and AT2 receptors. Clin Sci (Lond) 121: 297-303

Catarata MJ, Ribeiro R, Oliveira MJ, Robalo Cordeiro C, Medeiros R (2020) Renin-Angiotensin System in Lung Tumor and Microenvironment Interactions. Cancers (Basel) 12

DeCaprio JA (2021) Molecular Pathogenesis of Merkel Cell Carcinoma. Annu Rev Pathol 16: 6991

Fan K, Spassova I, Gravemeyer J, Ritter C, Horny K, Lange A, Gambichler T, Odum N, Schrama D, Schadendorf D et al (2021) Merkel cell carcinoma-derived exosome-shuttle miR-375 induces fibroblast polarization by inhibition of RBPJ and p53. Oncogene 40: 980-996

Feng H, Shuda M, Chang Y, Moore PS (2008) Clonal integration of a polyomavirus in human Merkel cell carcinoma. Science 319: 1096-1100

George AJ, Thomas WG, Hannan RD (2010) The renin-angiotensin system and cancer: old dog, new tricks. Nat Rev Cancer 10: 745-759

Goh G, Walradt T, Markarov V, Blom A, Riaz N, Doumani R, Stafstrom K, Moshiri A, Yelistratova L, Levinsohn J et al (2016) Mutational landscape of MCPyV-positive and MCPyVnegative Merkel cell carcinomas with implications for immunotherapy. Oncotarget 7: 3403-3415

Gonzalez-Vela MDC, Curiel-Olmo S, Derdak S, Beltran S, Santibanez M, Martinez N, CastilloTrujillo A, Gut M, Sanchez-Pacheco R, Almaraz C et al (2017) Shared Oncogenic Pathways Implicated in Both Virus-Positive and UV-Induced Merkel Cell Carcinomas. J Invest Dermatol 137: 197-206 
Gugliesi F, Mondini M, Ravera R, Robotti A, de Andrea M, Gribaudo G, Gariglio M, Landolfo S (2005) Up-regulation of the interferon-inducible IFI16 gene by oxidative stress triggers p53 transcriptional activity in endothelial cells. J Leukoc Biol 77: 820-829

Hafner C, Houben R, Baeurle A, Ritter C, Schrama D, Landthaler M, Becker JC (2012) Activation of the PI3K/AKT pathway in Merkel cell carcinoma. PLoS One 7: e31255

Harms KL, Healy MA, Nghiem P, Sober AJ, Johnson TM, Bichakjian CK, Wong SL (2016) Analysis of Prognostic Factors from 9387 Merkel Cell Carcinoma Cases Forms the Basis for the New 8th Edition AJCC Staging System. Ann Surg Oncol 23: 3564-3571

Harms PW, Vats P, Verhaegen ME, Robinson DR, Wu YM, Dhanasekaran SM, Palanisamy N, Siddiqui J, Cao X, Su F et al (2015) The Distinctive Mutational Spectra of Polyomavirus-Negative Merkel Cell Carcinoma. Cancer Res 75: 3720-3727

Heichler C, Scheibe K, Schmied A, Geppert CI, Schmid B, Wirtz S, Thoma OM, Kramer V, Waldner MJ, Buttner C et al (2020) STAT3 activation through IL-6/IL-11 in cancer-associated fibroblasts promotes colorectal tumour development and correlates with poor prognosis. Gut 69: $1269-1282$

Hetheridge C, Mavria G, Mellor H (2011) Uses of the in vitro endothelial-fibroblast organotypic co-culture assay in angiogenesis research. Biochem Soc Trans 39: 1597-1600

Hinshaw DC, Shevde LA (2019) The Tumor Microenvironment Innately Modulates Cancer Progression. Cancer Res 79: 4557-4566

Kalluri R (2016) The biology and function of fibroblasts in cancer. Nat Rev Cancer 16: 582-598

Karakasheva TA, Lin EW, Tang Q, Qiao E, Waldron TJ, Soni M, Klein-Szanto AJ, Sahu V, Basu D, Ohashi S et al (2018) IL-6 Mediates Cross-Talk between Tumor Cells and Activated Fibroblasts in the Tumor Microenvironment. Cancer Res 78: 4957-4970

Knips J, Czech-Sioli M, Spohn M, Heiland M, Moll I, Grundhoff A, Schumacher U, Fischer N (2017) Spontaneous lung metastasis formation of human Merkel cell carcinoma cell lines transplanted into scid mice. Int J Cancer 141: 160-171

Lau EY, Lo J, Cheng BY, Ma MK, Lee JM, Ng JK, Chai S, Lin CH, Tsang SY, Ma S et al (2016) Cancer-Associated Fibroblasts Regulate Tumor-Initiating Cell Plasticity in Hepatocellular Carcinoma through c-Met/FRA1/HEY1 Signaling. Cell Rep 15: 1175-1189

Leung CS, Yeung TL, Yip KP, Wong KK, Ho SY, Mangala LS, Sood AK, Lopez-Berestein G, Sheng J, Wong ST et al (2018) Cancer-associated fibroblasts regulate endothelial adhesion protein LPP to promote ovarian cancer chemoresistance. J Clin Invest 128: 589-606

Love MI, Huber W, Anders S (2014) Moderated estimation of fold change and dispersion for RNAseq data with DESeq2. Genome Biol 15: 550

Marchio S, Lahdenranta J, Schlingemann RO, Valdembri D, Wesseling P, Arap MA, Hajitou A, Ozawa MG, Trepel M, Giordano RJ et al (2004) Aminopeptidase A is a functional target in angiogenic blood vessels. Cancer Cell 5: 151-162

Mendoza MD, Santonja C, Gonzalez-Vela C, Concha A, Iglesias Pena N, Andres-Esteban EM, Vaque JP, Cereceda L, Pajares R, Kutzner H et al (2020) The presence of Merkel cell carcinoma 
polyomavirus is associated with a distinct phenotype in neoplastic Merkel cell carcinoma cells and their tissue microenvironment. PLoS One 15: e0232517

Nanus DM, Engelstein D, Gastl GA, Gluck L, Vidal MJ, Morrison M, Finstad CL, Bander NH, Albino AP (1993) Molecular cloning of the human kidney differentiation antigen gp160: human aminopeptidase A. Proc Natl Acad Sci U S A 90: 7069-7073

Nardi V, Song Y, Santamaria-Barria JA, Cosper AK, Lam Q, Faber AC, Boland GM, Yeap BY, Bergethon K, Scialabba VL et al (2012) Activation of PI3K signaling in Merkel cell carcinoma. Clin Cancer Res 18: 1227-1236

Nehmann N, Wicklein D, Schumacher U, Muller R (2010) Comparison of two techniques for the screening of human tumor cells in mouse blood: quantitative real-time polymerase chain reaction (qRT-PCR) versus laser scanning cytometry (LSC). Acta Histochem 112: 489-496

Paulson KG, Iyer JG, Simonson WT, Blom A, Thibodeau RM, Schmidt M, Pietromonaco S, Sokil M, Warton EM, Asgari MM et al (2014) CD8+ lymphocyte intratumoral infiltration as a stageindependent predictor of Merkel cell carcinoma survival: a population-based study. Am J Clin Pathol 142: 452-458

Pinter M, Jain RK (2017) Targeting the renin-angiotensin system to improve cancer treatment: Implications for immunotherapy. Sci Transl Med 9

Reaux A, Fournie-Zaluski MC, Llorens-Cortes C (2001) Angiotensin III: a central regulator of vasopressin release and blood pressure. Trends Endocrinol Metab 12: 157-162

Rosen ST, Gould VE, Salwen HR, Herst CV, Le Beau MM, Lee I, Bauer K, Marder RJ, Andersen R, Kies MS et al (1987) Establishment and characterization of a neuroendocrine skin carcinoma cell line. Lab Invest 56: 302-312

Samimi M, Benlalam H, Aumond P, Gaboriaud P, Fradin D, Kervarrec T, Florenceau L, Vignard V, Blom A, Touze A et al (2019) Viral and tumor antigen-specific CD8 T-cell responses in Merkel cell carcinoma. Cell Immunol 344: 103961

Saraswati S, Marrow SMW, Watch LA, Young PP (2019) Identification of a pro-angiogenic functional role for FSP1-positive fibroblast subtype in wound healing. Nat Commun 10: 3027

Schindelin J, Arganda-Carreras I, Frise E, Kaynig V, Longair M, Pietzsch T, Preibisch S, Rueden C, Saalfeld S, Schmid B et al (2012) Fiji: an open-source platform for biological-image analysis. Nat Methods 9: 676-682

Strimmer K (2008) fdrtool: a versatile R package for estimating local and tail area-based false discovery rates. Bioinformatics 24: 1461-1462

Trinidad CM, Torres-Cabala CA, Prieto VG, Aung PP (2019) Update on eighth edition American Joint Committee on Cancer classification for Merkel cell carcinoma and histopathological parameters that determine prognosis. J Clin Pathol 72: 337-340

Uemura H, Ishiguro H, Ishiguro Y, Hoshino K, Takahashi S, Kubota Y (2008) Angiotensin II induces oxidative stress in prostate cancer. Mol Cancer Res 6: 250-258 
Wen S, Hou Y, Fu L, Xi L, Yang D, Zhao M, Qin Y, Sun K, Teng Y, Liu M (2019) Cancerassociated fibroblast (CAF)-derived IL32 promotes breast cancer cell invasion and metastasis via integrin beta3-p38 MAPK signalling. Cancer Lett 442: 320-332

Wright JW, Mizutani S, Harding JW (2012) Focus on Brain Angiotensin III and Aminopeptidase A in the Control of Hypertension. Int J Hypertens 2012: 124758

Yu G, Wang LG, Han Y, He QY (2012) clusterProfiler: an R package for comparing biological themes among gene clusters. OMICS 16: 284-287

Zhao N, Peacock SO, Lo CH, Heidman LM, Rice MA, Fahrenholtz CD, Greene AM, Magani F, Copello VA, Martinez MJ et al (2019) Arginine vasopressin receptor 1a is a therapeutic target for castration-resistant prostate cancer. Sci Transl Med 11

Zini S, Fournie-Zaluski MC, Chauvel E, Roques BP, Corvol P, Llorens-Cortes C (1996) Identification of metabolic pathways of brain angiotensin II and III using specific aminopeptidase inhibitors: predominant role of angiotensin III in the control of vasopressin release. Proc Natl Acad Sci U S A 93: 11968-11973 


\section{Figure Legends}

\section{Figure 1. Characterization of MCC-derived cancer associated fibroblasts (CAFs).}

A Immunoblot analysis on total cell lysates from the isolated CAFs along with a panel of control cell lines [human foreskin fibroblasts (HFFs); mouse myofibroblasts, C2C12; immortalized human keratinocytes, N/TERT; human umbilical vein endothelial cells (HUVECs)] probed with antibodies against vimentin, $\alpha$ SMA-1, CK14, CD31, and $\beta$-actin. Representative blots from triplicate experiments are shown.

B Collagen contractility assay evaluated as percentage of gel contraction in gel without cells (-), and gel containing normal fibroblasts (HFFs) or individual CAFs (rPt2-pPt9); representative pictures are shown on the top of the box plot graph $(n=3)$.

C qRT-PCR analysis for IL-8, IL-6, CXCL12, and TGF- $\beta 1$ mRNA expression levels in total RNA extracts from CAFs or HFFs. Values were normalized to GAPDH mRNA and plotted as fold induction over HFFs $(n=4)$.

Data Information: In (B), the whisker-box plots represent the $25^{\text {th }}-75^{\text {th }}$ percentiles, with midlines indicating the median values and whiskers extending to the minimum and maximum values. In (C), values are represented as mean \pm SD. $n$ indicates the number of independent experiments. In (B-C), the significance levels are as follows: ${ }^{*} P<0.05, * * P<0.01$, $* * * P<0.001$ vs HFFs; classical oneway ANOVA followed by post-hoc Bonferroni correction.

\section{Figure 2. Characterization of the tumor microenvironment in MCC patients.}

A Representative images of hematoxylin and eosin (H\&E) staining and immunohistochemical staining (brown) in the primary MCC from Pt5 (upper row) and Pt6 (lower row). Serial tissue sections were stained with antibodies against vimentin, $\alpha$ SMA-1, CD8, and CD68, respectively. Scale bars: $2 \mathrm{~mm}$. Black squares insets show 25 x magnification; scale bar: 100 $\mu \mathrm{m}$. 
B The intratumoral stromal streaks in the original MCC from the study cohort-primary and relapsed when available_were quantified with a pixel classifier trained in QuPath. The streaks are represented as pie charts with stromal components (dark grey) given as percentages (upper row). Pie chart and scatter plots showing the number of cells per $\mathrm{mm}^{2}$ that stained for $\alpha$ SMA$1^{+}$(green), $\mathrm{CD}^{+}$(red), and $\mathrm{CD}^{+} 8^{+}$(blue) in the intratumoral stromal streaks (lower raw). The number of cells $/ \mathrm{mm}^{2}$ was quantified from 10 independent tumor areas (represented as individual point) using the fast cell count tool by QuPath.

Figure 3. Patient-derived CAFs significantly enhance MKL-1 xenograft tumor growth and metastasis formation into SCID mice.

A The xenograft tumors grown into SCID mice at the endpoint of the experiment (three months) are shown from three individual mice, with the exception of rPt5 CAFs $(n=2)$, obtained by injecting MKL-1 cells alone (-) or with $10^{6}$ HFFs or CAFs (rPt2 to pPt9) into SCID mice. Images of the explanted xenografts are shown $(n=3$ for each combination, with the sole exception of rPt5 $n=2$ ).

B Tumor growth was weekly assessed, and growth curves are represented as mean \pm SD for each group. Statistical analysis of MKL-1/CAFs (rPt2 to pPt9) was performed by two-way ANOVA vs either MKL-1 alone (-) or MKL-1/HFF curves. $P$-values, when significant, are indicated in the table.

C Tumor weight in each tumor was evaluated at 3 months after injection.

D Quantification of metastatic cell number in lungs, brain, and bone marrow of engrafted mice as determined by real-time qPCR for human Alu sequences. The number of cancer cells was normalized to the input mouse DNA ng.

Data Information: In $(C, D)$, results are represented as mean values of the different mouse replicates; each symbol represents an individual animal. Statistical analysis was performed by classical one-way ANOVA followed by post-hoc Bonferroni correction. Significance values of 
MKL-1/CAFs (rPt2 to pPt9) vs MKL-1/HFFs: $* P<0.05, * * P<0.01, * * * P<0.001$ or $v s$ MKL-1: ${ }^{\#} P<0.05,{ }^{\#} P<0.01,{ }^{\# \#} P<0.001$.

\section{Figure 4. MCC-derived CAFs potentiate angiogenesis in MKL-1 xenografts.}

A Representative images of serial tissue sections from FFPE blocks of the xenografts obtained by subcutaneous injection of MKL-1 cells alone (-), MKL-1 plus normal fibroblasts (HFFs), or MKL-1 plus rPt3, pPt9, pPt4, or rPt5 CAFs. Tissue samples were stained with antibodies against human vimentin (left panels) or CD31 (central column). The right panels show the immunofluorescence analysis of serial sections co-stained with anti-vimentin antibodies as follows: human-specific (h-vim, green) and anti-human/mouse (h/m-vim, red). The nuclei were counterstained with DAPI (blue). The area shown in the central and right panels is a magnification of the area contained in the respective hollow rectangle. Vessels are highlighted with arrows.

B The number of $\mathrm{h}$-vim ${ }^{+}$cells $/ \mathrm{mm}^{2}$ was measured in the entire tumor mass sections of all xenografts using the fast cell count tool from QuPath software.

C Vessel density in sections stained with antibodies against CD31. Microvessels were counted manually in at least 8-10 fields per tumor section at $\times 200$ magnification.

D Vessel size was determined by measuring the wider vessel diameter with QuPath software.

E Correlation of vessel diameter with number of CAFs found in close proximity to vessels. Vessel quantification was performed as described in (D) and a score of 1 (low density, light gray), 2 (medium density, dark gray), and 3 (high density, black) was given based on the density of hvim $^{+}$cells surrounding the vessels. The number of blood vessels, scored 1 to 3 in each explanted tumor, is represented as the average percentage within the replicates ( $n=3$ for each combination, with the sole exception of MKL-1/rPt5CAFs, $n=2$ ).

F For each blood vessel, regardless of the mouse group, the size (y-axes) is plotted against its corresponding score (x-axes). 
Data Information: in (B) data are represented as mean $\pm \mathrm{SD}$, and each dot represents an individual animal; In (C and D), the whisker-box plots represent the $25^{\text {th }}-75^{\text {th }}$ percentiles, with midlines indicating the median values, whiskers extending to the $10^{\text {th }}-90^{\text {th }}$ percentiles, and dots showing outliers. Significant levels are $* P<0.05, * * P<0.01$, ***P $<0.001 v s$ MKL-1/HFFs or ${ }^{\#} P<0.05$, \#\# $P<0.01,{ }^{\# \# \#} P<0.001$ vs MKL-1 in (B, C, and D) and $* * * P<0.001$ in $(\mathrm{F})$ from classical one-way ANOVA followed by post-hoc Bonferroni correction.

Figure 5. Differential gene expression profiling of xenografts injected with MKL-1/CAFs from rPt2, pPt4, and 5 vs those injected with MKL1/HFFs or MKL-1 alone.

A Heatmap of RNA-Seq data showing genes resulting differentially expressed $(\log 2 \mathrm{FC} \mid>1, P$ adj $<0.01$ ) between tumor CAF (MKL-1/CAFs from rPt2, pPt4, and rPt5) and CTRL (MKL-1 and MKL-1/HFFs) groups.

B Volcano plot of RNA-Seq data showing up- and downregulated genes in MKL-1/CAFs vs MKL-1/CTRLs. Significantly deregulated genes (DEG; $\log 2 \mathrm{FC} \mid>1, P$ adj $<0.01$ ) are visualized as red dots. The vertical bars delimitate $\log 2 \mathrm{FC} \mid 1$, while the horizontal ones delimitate $-\log 10$ padj $=1.3$.

C GO term analysis. DEG genes resulting from CAFs vs CTRLs were analyzed using the DAVID bioinformatics resources. Circle size maps the number of genes in each GOterm/KEGG pathway, whereas colors map significance ( $P$ adjust).

D KEGG pathway enrichment analysis. This analysis was performed as described above.

Figure 6. AVPR1A, ENPEP and MME is differentially expressed by fast-growing xenografts.

A qRT-PCR analysis for ENPEP, MEE and AVPR1A mRNA expression levels in total RNA extracts from individual explanted xenografts. Values were normalized to GAPDH and plotted as fold induction over MKL-1 plus HFF-injected tumors. Data are represented as means $\pm \mathrm{SD}$, and each dot represents an individual animal. Statistical analysis was performed by one-way 
ANOVA followed by post-hoc Bonferroni correction. Significant levels: $* P<0.05$, *** $P<$ 0.001 vs MKL-1/HFFs or ${ }^{\#} P<0.05,{ }^{\# \#} P<0.01$ vs MKL-1.

B Spearman rank correlation of $\mathrm{h}$-vim ${ }^{+}$cell/ $/ \mathrm{mm}^{2}$ (y-axes) with ENPEP, MEE and AVPR1A mRNA expression levels (x-axes). p ( $P$-value) and $\mathrm{r}$ (spearman correlation coefficient) are indicated in the graph.

C qRT-PCR analysis for ENPEP and MME mRNA expression levels from CAFs, HFFs, or MKL-1. Values were normalized to GAPDH and plotted as fold induction over HFFs. Data are represented as means \pm SD. Statistical analysis was performed by one-way ANOVA followed by post-hoc Bonferroni correction. Significant levels: $* P<0.05, * * P<0.01$ vs HFFs.

Figure 7. Pharmacological inhibition of the $\mathrm{APA}_{\mathbf{A}} \mathrm{AT}_{1} \mathbf{R}$ pathway impairs CAF-induced tubulogenesis.

A Representative confocal images of HDBECs co-cultured with rPt5 CAFs for 10 days in the presence of amastatin or vehicle as control $(\mathrm{MeOH})$ at the indicated concentrations. Scale bar $=$ $100 \mu \mathrm{m}$.

B Quantification of mean tubule length (left panel), total tubule length (central panel), or tubule density (right panel) from the co-cultures shown in panel A. Data represent mean \pm SD of three independent experiments, one-way ANOVA followed by post-hoc Bonferroni correction. Significant levels: $* P<0.05, * * P<0.01, * * * P<0.001$.

C Representative confocal images of HDBECs co-cultured with rPt5 CAFs for 10 days in the presence of candesartan $(10 \mu \mathrm{M})$ or vehicle as control $(\mathrm{DMSO})$. Scale bar $=100 \mu \mathrm{m}$.

D Quantification of mean tubule length (left panel), total tubule length (central panel), or tubule density (right panel) from the co-cultures shown in panel C. Data represent mean \pm SD of three independent experiments, unpaired $t$ test; $* P<0.05$, $* * P<0.01$. 
bioRxiv preprint doi: https://doi.org/10.1101/2021.07.30.454425; this version posted August 3, 2021. The copyright holder for this preprint (which was not certified by peer review) is the author/funder. All rights reserved. No reuse allowed without permission.

Table 1. Clinicopathological features of the MCC cohort

Table 2. Top 10 overexpressed genes in xenograft tumors derived from mice engrafted with MKL-1/rPt2, pPt4, or rPt5 CAFs compared to controls (MKL-1 and MKL-1/HFFs) 


\section{Expanded View Figure Legends}

Figure EV1. Characterization of the stromal components. Scatter plots showing the number of cells per $\mathrm{mm}^{2}$ that were $\alpha \mathrm{SMA}^{+}{ }^{+}$(green), $\mathrm{CD}^{+}$(red), and $\mathrm{CD}^{+} 8^{+}$(blue) in the tumor edges (A) and nests (B). The number of cells $/ \mathrm{mm}^{2}$ was quantified from 10 independent tumor areas (represented as individual points) using the fast cell count tool by QuPath.

Figure EV2. Tumor growth assessment. Tumor growth curve of each mice group co-injected with MKL-1 and CAFs (rPt2 to pPt9) compared to mice injected with MKL-1 cells alone (black lines) or with MKL-1 plus HFFs (grey lines). Values at each time point are represented as mean \pm SD within the mouse replicates for each group. Differences between curves were evaluated by two-way ANOVA. Statistical analysis at each time point was performed by two-way ANOVA followed posthoc Bonferroni correction. ${ }^{*} P<0.05$, ${ }^{*} P<<0.01$, *** $P<0.001$ vs MKL-1/HFF tumors or ${ }^{\#} P<$ $0.05,{ }^{\# \#} P<0.01,{ }^{\# \#} P<0.001$ vs MKL-1 tumors.

Figure EV3. Ingenuity pathway analysis. This analysis shows different diseases and functions predicted to be activated (z-score $>2$, orange bars) or inhibited (z-score $<2$, blue bars) in MKL1/CAFs vs MKL-1/CTRLs.

Figure EV4. CAFs support angiogenesis in an in vitro model of tubulogenesis. CAFs or HFF were co-cultured with HDBEC for 10 days. Representative images of CD31 immunostaining of the tubule-like structures (red). Nuclei were counterstained with DAPI (blue). Scale bars $100 \mu \mathrm{m}$.

Figure EV5. Lack of enhanced or synergistic effects upon co-treatment with amastatin and candesartan. rPt5 CAFs were co-cultured with HDBECs for 10 days in the presence of amastatin, candesartan, the combination of both inhibitors, or vehicle alone (MeOH for amastatin and DMSO 
for candesartan, respectively) at the indicated concentrations. Panel A shows representative images of CD31 immunostaining of the tubule-like structures (red). Nuclei were counterstained with DAPI (blue). Scale bars $100 \mu \mathrm{m}$. Panel B shows the quantification of mean tubule length using Fiji software. Panel $\mathrm{C}$ shows the quantification of the total tubule length as measured by WimTube software. Panel D shows the tubule density as measured by WimTube software. Statistical analysis was performed by classical one-way ANOVA followed by Bonferroni correction. ${ }^{*} P<0.05$, $* * P<$ $0.01, * * * P<0.001$ vs vehicle control. 

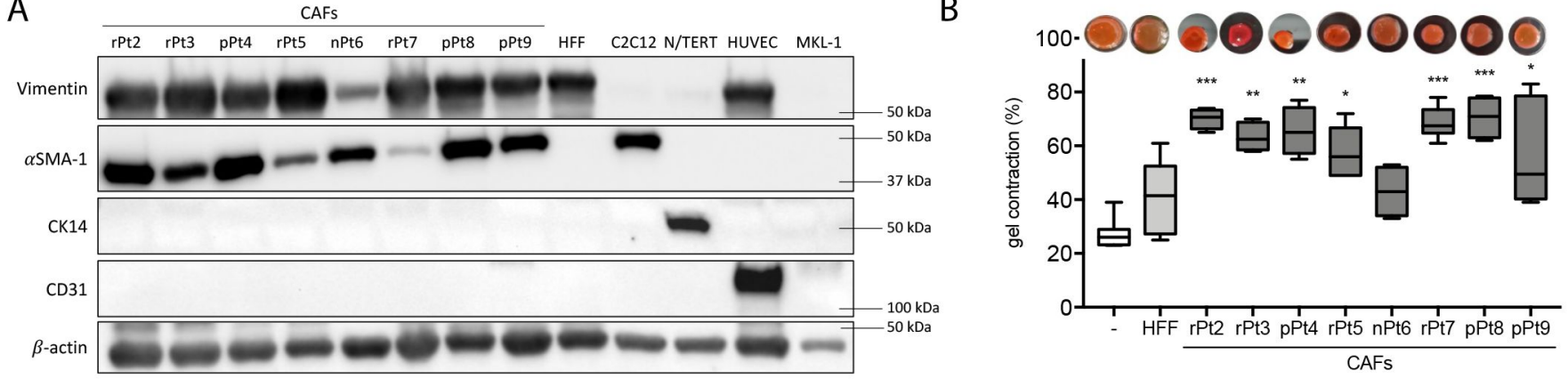

C
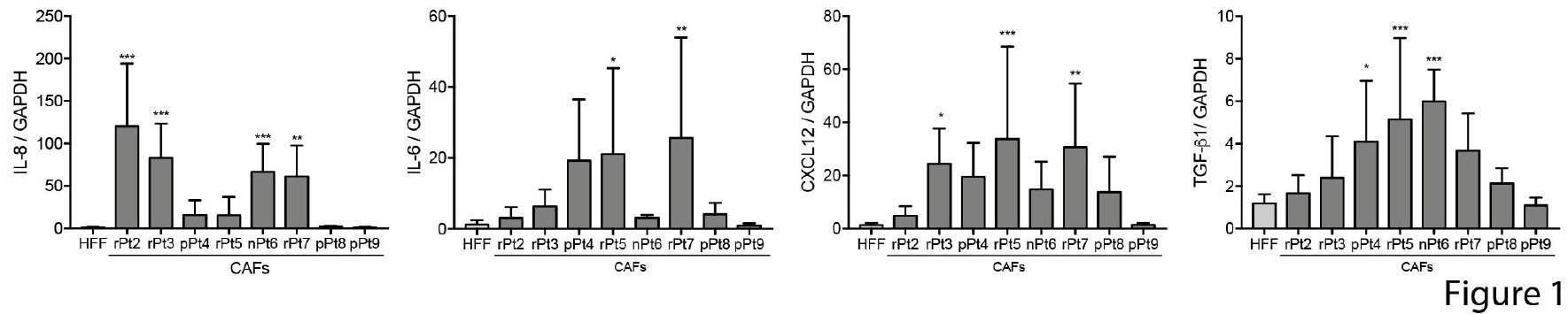


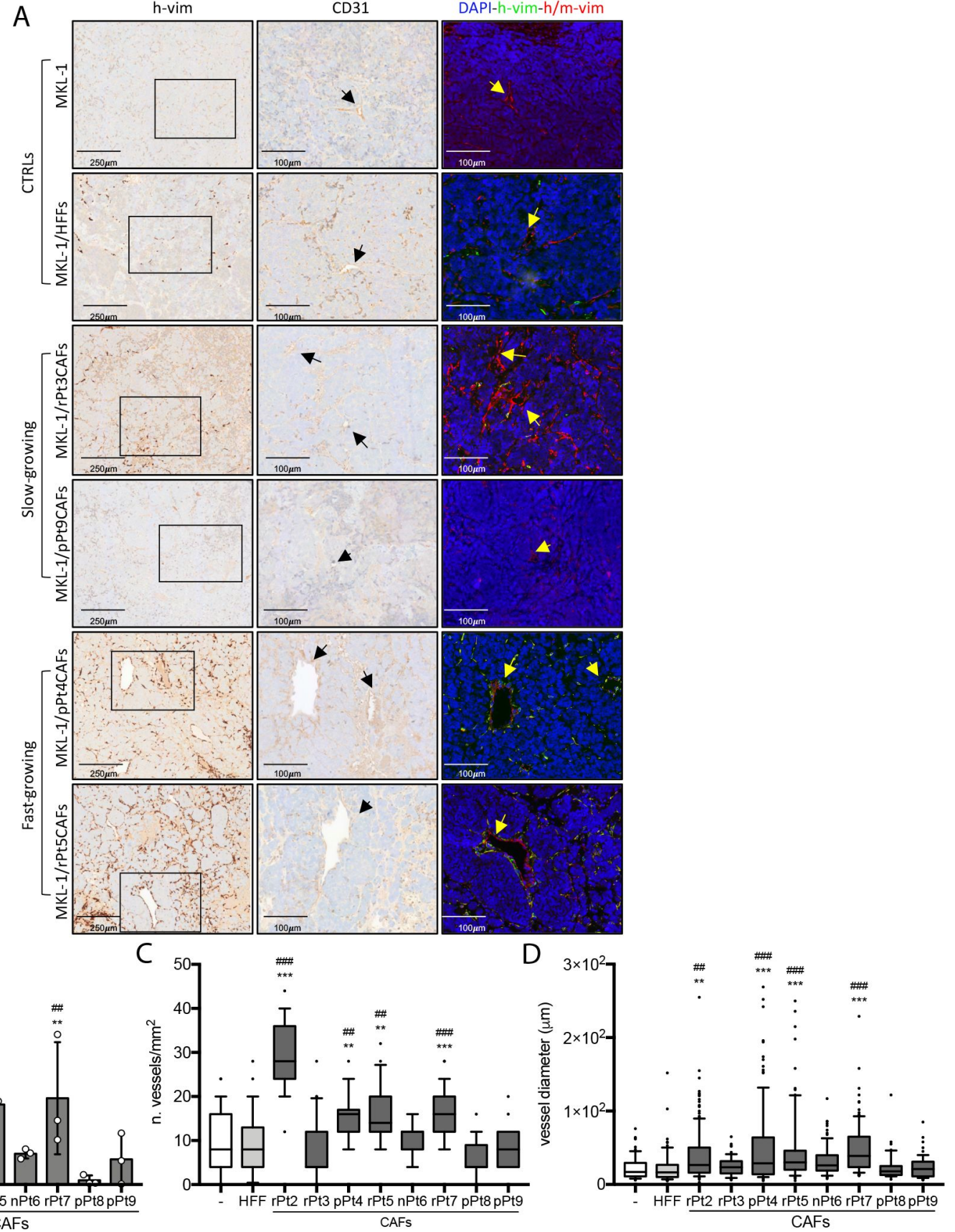

E

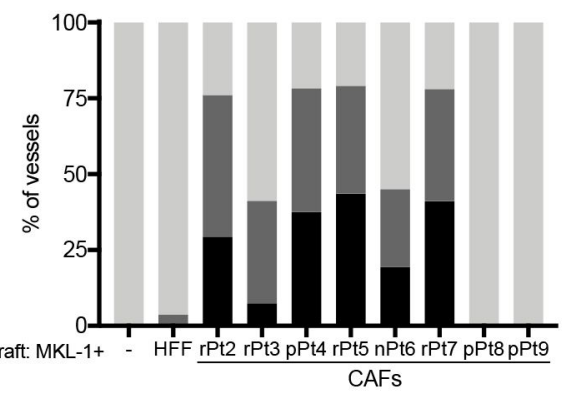

$\mathrm{F}$

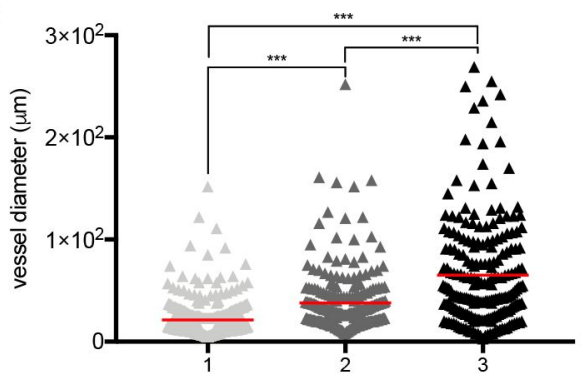

Figure 4 


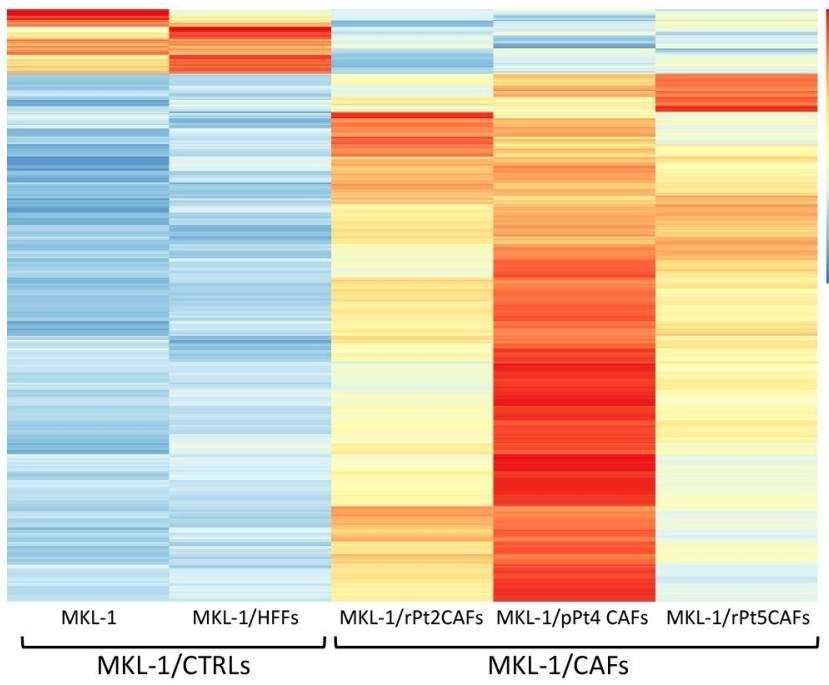

C

\section{GO_terms}

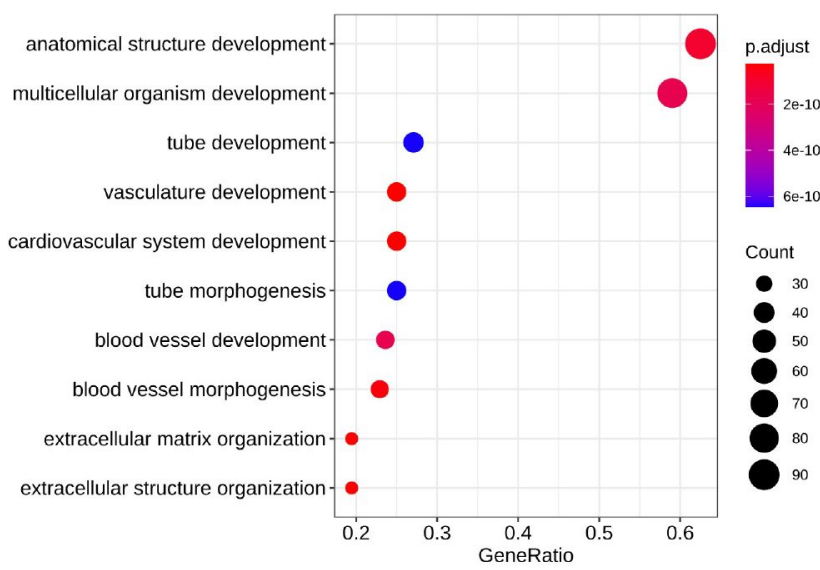

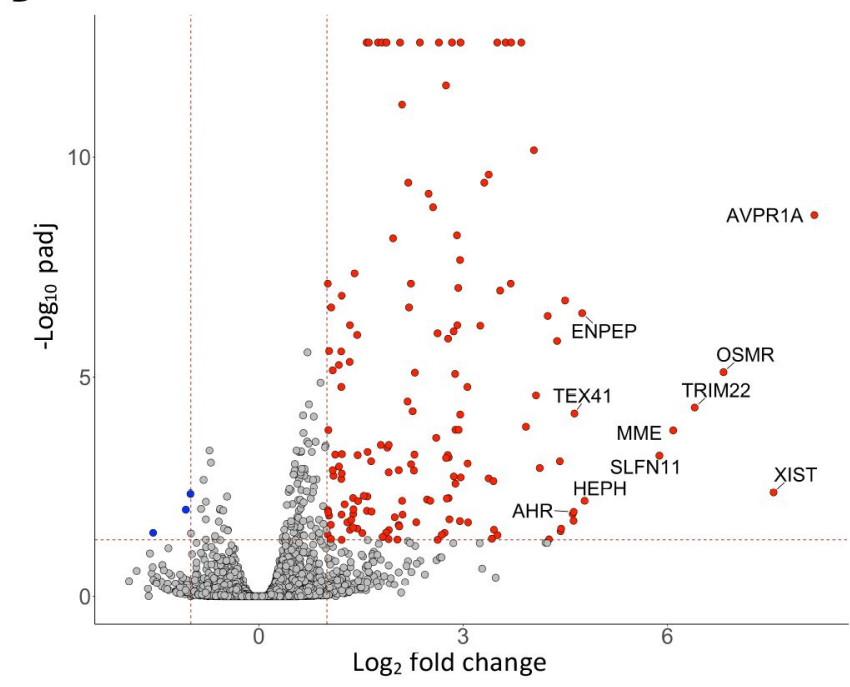

D

\section{KEGG pathways}

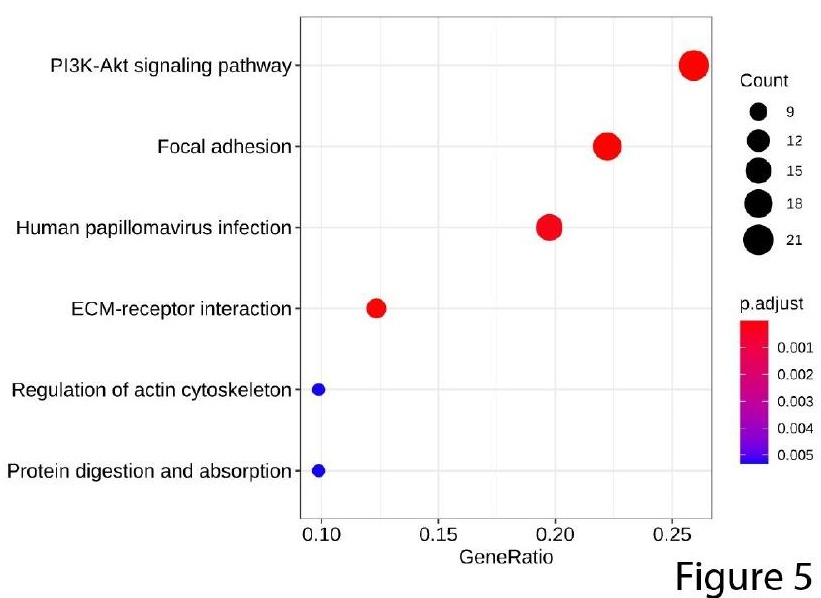




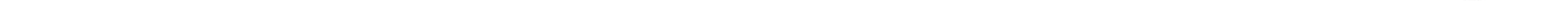




\section{Table 1. Clinicopathological features of the MCC cohort}

\begin{tabular}{|c|c|c|c|c|c|c|c|}
\hline Patient & $\begin{array}{l}\text { Gender/Birth-Death } \\
\text { (cause of death) }\end{array}$ & Date of surgery & & & Site & $\begin{array}{l}\text { MCPyV status* } \\
\text { (viral load) }\end{array}$ & Isolated CAFs \\
\hline \multirow{2}{*}{1} & \multirow{2}{*}{$\begin{array}{l}F / 1936-2017 \\
(+U C)\end{array}$} & $\begin{array}{c}\text { Jul } 2015 \\
\text { (Primary MCC) }\end{array}$ & 1 & $\begin{array}{l}\text { T1 } \\
\text { No } \\
\text { Mo }\end{array}$ & \multirow{2}{*}{$\begin{array}{l}\text { Buttock } \\
\text { (Dermal) }\end{array}$} & \multirow{2}{*}{$\stackrel{+}{+}$} & \\
\hline & & $\begin{array}{c}\text { Sep } 2015 \\
\text { (Local recurrence MCC) }\end{array}$ & $\| \mathrm{A}$ & $\begin{array}{l}\text { T3 } \\
\text { No } \\
\text { Mo } \\
\end{array}$ & & & rPt1 (failed) \\
\hline \multirow{2}{*}{2} & \multirow{2}{*}{$\begin{array}{l}M / 1937-2017 \\
\text { (+UC) }\end{array}$} & $\begin{array}{c}\text { Set } 2016 \\
\text { (Primary MCC) }\end{array}$ & 1 & $\begin{array}{l}\text { T1 } \\
\text { No } \\
\text { Mo }\end{array}$ & \multirow{2}{*}{$\begin{array}{c}\text { Face } \\
\text { (Epidermal) }\end{array}$} & \multirow{2}{*}{$\begin{array}{l}+ \\
(4)\end{array}$} & \\
\hline & & $\begin{array}{c}\text { Feb } 2017 \\
\text { (Local recurrence MCC) }\end{array}$ & $\| \mathrm{A}$ & $\begin{array}{l}\text { T2 } \\
\text { No } \\
\text { Mo }\end{array}$ & & & rPt2 \\
\hline \multirow{3}{*}{3} & \multirow{3}{*}{$\begin{array}{l}M / 1932-2018 \\
(+M C C)\end{array}$} & $\begin{array}{c}\text { Feb } 2017 \\
\text { (Primary MCC) }\end{array}$ & $\| \mathrm{A}$ & $\begin{array}{l}\text { T2 } \\
\text { No } \\
\text { MO }\end{array}$ & $\begin{array}{c}\text { Face } \\
\text { (Epidermal) }\end{array}$ & \multirow{3}{*}{-} & \\
\hline & & $\begin{array}{c}\text { Apr } 2017 \\
\text { (Local recurrence MCC) }\end{array}$ & $\| \mathrm{A}$ & $\begin{array}{l}\text { T2 } \\
\text { No } \\
\text { Mo } \\
\end{array}$ & $\begin{array}{c}\text { Face } \\
\text { (Epidermal) }\end{array}$ & & rPt3 \\
\hline & & $\begin{array}{c}\text { Aug } 2017 \\
\text { (Metastasis) }\end{array}$ & IV & $\begin{array}{l}\text { N3 } \\
\text { M1 }\end{array}$ & Brain & & \\
\hline 4 & $\begin{array}{c}F / 1929-2018 \\
(+\cup C)\end{array}$ & $\begin{array}{c}\text { Jul } 2017 \\
\text { (Primary MCC) } \\
\end{array}$ & IIA & $\begin{array}{l}\text { T2 } \\
\text { No } \\
\text { M0 } \\
\end{array}$ & $\begin{array}{c}\text { Leg } \\
\text { (Epidermal) } \\
\end{array}$ & - & pPt4 \\
\hline \multirow{3}{*}{5} & \multirow{3}{*}{$F / 1939$} & $\begin{array}{c}\text { Dec } 2016 \\
\text { (Primary MCC) }\end{array}$ & IIIA & $\begin{array}{l}\text { T2 } \\
\text { N1a } \\
\text { Mo } \\
\end{array}$ & \multirow{3}{*}{$\begin{array}{c}\text { Arm } \\
\text { (Dermal) }\end{array}$} & \multirow{3}{*}{$\begin{array}{l}+ \\
(6)\end{array}$} & \\
\hline & & $\begin{array}{c}\text { Aug } 2017 \\
\text { (Local recurrence MCC) }\end{array}$ & IIIA & $\begin{array}{l}\text { T2 } \\
\text { N1a } \\
\text { MO }\end{array}$ & & & rPt5 \\
\hline & & $\begin{array}{c}\text { Mar } 2018 \\
\text { (In transit metastasis) }\end{array}$ & IIIB & $\begin{array}{l}\text { T2 } \\
\text { N2 } \\
\text { M0 } \\
\end{array}$ & & & \\
\hline \multirow[b]{2}{*}{6} & \multirow[b]{2}{*}{$F / 1942$} & $\begin{array}{c}\text { Mar } 2017 \\
\text { (Primary MCC) }\end{array}$ & 1 & $\begin{array}{l}\text { T1 } \\
\text { No } \\
\text { M0 }\end{array}$ & $\begin{array}{l}\text { Forehead } \\
\text { (Dermal) }\end{array}$ & \multirow{2}{*}{$\stackrel{+}{+}$} & \\
\hline & & $\begin{array}{l}\text { Dec } 2017 \\
\text { (Lymph node } \\
\text { metastasis) }\end{array}$ & IIIB & $\begin{array}{l}\mathrm{T} 1 \\
\text { N1b } \\
\text { M0 }\end{array}$ & Head and neck & & nPt6 \\
\hline \multirow{2}{*}{7} & \multirow{2}{*}{$F / 1930$} & $\begin{array}{c}\text { Jul } 2017 \\
\text { (Primary MCC) }\end{array}$ & 1 & $\begin{array}{l}\text { T1 } \\
\text { No } \\
\text { Mo }\end{array}$ & \multirow{2}{*}{$\begin{array}{c}\text { Arm } \\
\text { (Dermal) }\end{array}$} & \multirow{2}{*}{ - } & \\
\hline & & $\begin{array}{c}\text { Apr } 2018 \\
\text { (Local recurrence) }\end{array}$ & 1 & $\begin{array}{l}\text { T1 } \\
\text { No } \\
\text { Mo } \\
\end{array}$ & & & rPt7 \\
\hline 8 & $F / 1932$ & $\begin{array}{c}\text { Aug } 2018 \\
\text { (Primary MCC) } \\
\end{array}$ & IIA & $\begin{array}{l}\text { T2 } \\
\text { No } \\
\text { MO } \\
\end{array}$ & $\begin{array}{c}\text { Leg } \\
\text { (Dermal) }\end{array}$ & $\begin{array}{c}+ \\
(12) \\
\end{array}$ & pPt8 \\
\hline 9 & $M / 1951$ & $\begin{array}{c}\text { Apr2019 } \\
\text { (Primary MCC) }\end{array}$ & IIA & $\begin{array}{l}\text { T3 } \\
\text { No } \\
\text { M0 }\end{array}$ & $\begin{array}{c}\text { Lumbosacral region } \\
\text { (Dermal) }\end{array}$ & $\begin{array}{c}+ \\
(2)\end{array}$ & pPt9 \\
\hline
\end{tabular}


Table 2. Top 10 overexpressed genes in xenograft tumors derived from mice engrafted with MKL-1/rPt2, pPt4, or rPt5 CAFs compared to controls (MKL-1 and MKL-1/HFFs)

\begin{tabular}{lllll} 
Ensembl & Gene Symbol Name & Log2fold Change & padjust \\
\hline ENSG00000166148 & AVPR1A & Arginine Vasopressin Receptor 1A & 8.158054016 & $2,09 \mathrm{E}-09$ \\
ENSG00000229807 & XIST & X Inactive Specific Transcript & 7.558623094 & 0.004189 \\
ENSG00000145623 & OSMR & Oncostatin-M-Specific Receptor Subunit Beta & 6.823932521 & $7.74 \mathrm{E}-06$ \\
\hline ENSG00000132274 & TRIM22 & Tripartite Motif Containing 22 & 6.39999662 & $4.93 \mathrm{E}-05$ \\
ENSG00000196549 & MME & Membrane Metalloendopeptidase & 6.084916028 & 0.000162 \\
ENSG00000172716 & SLFN11 & Schlafen Family Member 11 & 5.882652425 & 0.000611 \\
ENSG00000089472 & HEPH & Hephaestin & 4.78388438 & 0.006487 \\
\hline ENSG00000138792 & ENPEP & Glutamyl Aminopeptidase & 4.747514005 & $3.53 \mathrm{E}-7$ \\
\hline ENSG00000226674 & TEX41 & Testis Expressed 41 & 4.634561309 & $6.75 \mathrm{E}-05$ \\
\hline ENSG00000106546 & AHR & Aryl Hydrocarbon Receptor & 4.623942417 & 0.011586 \\
\hline
\end{tabular}

